OPEN ACCESS

Edited by:

Rizwan Romee,

Dana-Farber Cancer Institute,

United States

Reviewed by:

Xin Yu,

Amgen, United States

Xuefeng Wang,

Soochow University, China

${ }^{*}$ Correspondence:

Yumin $L$

liym@/zu.edu.cn

Specialty section:

This article was submitted to

Cancer Immunity and Immunotherapy,

a section of the journal

Frontiers in Immunology

Received: 30 March 2021

Accepted: 04 May 2021

Published: 19 May 2021

Citation:

Miao L, Zhang Z, Ren Z, Tang F and

Li Y (2021) Obstacles and Coping

Strategies of CAR-T Cell Immunotherapy in Solid Tumors.

Front. Immunol. 12:687822.

doi: 10.3389/fimmu.2021.687822

\section{Obstacles and Coping Strategies of CAR-T Cell Immunotherapy in Solid Tumors}

\author{
Lele Miao ${ }^{1,2}$, Zhengchao Zhang ${ }^{1,2}$, Zhijian Ren ${ }^{1,2}$, Futian Tang $^{2}$ and Yumin $L i^{1,2^{*}}$ \\ ${ }^{1}$ Department of General Surgery, Second Hospital of Lanzhou University, Lanzhou, China, ${ }^{2}$ Key Laboratory of the Digestive \\ System Tumors of Gansu Province, Second Hospital of Lanzhou University, Lanzhou, China
}

Chimeric antigen receptor (CAR) T-cell immunotherapy refers to an adoptive immuno therapy that has rapidly developed in recent years. It is a novel type of treatment that enables $T$ cells to express specific CARs on their surface, then returns these T cells to tumor patients to kill the corresponding tumor cells. Significant strides in CAR-T cell immunotherapy against hematologic malignancies have elicited research interest among scholars in the treatment of solid tumors. Nonetheless, in contrast with the efficacy of CAR-T cell immunotherapy in the treatment of hematologic malignancies, its general efficacy against solid tumors is insignificant. This has been attributed to the complex biological characteristics of solid tumors. CAR-T cells play a better role in solid tumors, for instance by addressing obstacles including the lack of specific targets, inhibition of tumor microenvironment (TME), homing barriers of CAR-T cells, differentiation and depletion of CAR-T cells, inhibition of immune checkpoints, trogocytosis of CAR-T cells, tumor antigen heterogeneity, etc. This paper reviews the obstacles influencing the efficacy of CAR-T cell immunotherapy in solid tumors, their mechanism, and coping strategies, as well as economic restriction of CAR-T cell immunotherapy and its solutions. It aims to provide some references for researchers to better overcome the obstacles that affect the efficacy of CAR-T cells in solid tumors.

Keywords: chimeric antigen receptor T cell, immunotherapy, obstacles, coping strategies, solid tumors 


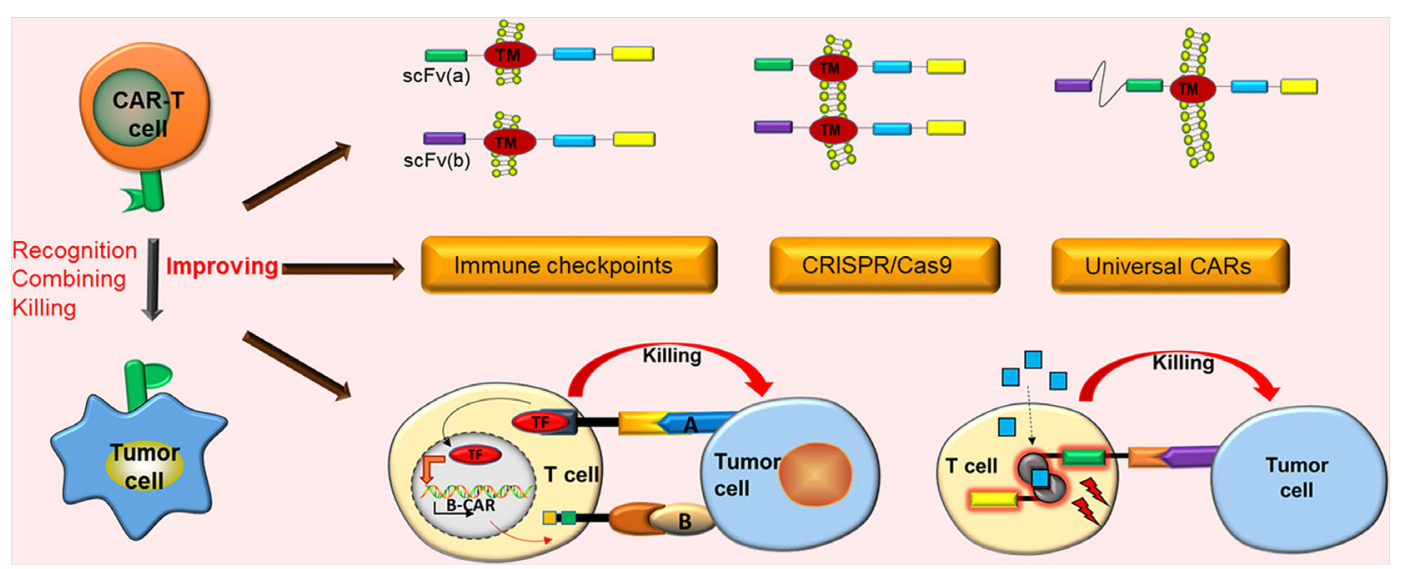

GRAPHICAL ABSTRACT | CAR-T cell immunotherapy has demonstrated notable future opportunities in the mainstream cancer treatment of solid tumors. However, it still has numerous obstacles in the treatment of solid tumors. Only by adopting effective strategies against these obstacles can CAR-T cells exert better curative effects in the treatment of solid tumors.

\section{INTRODUCTION}

Malignant tumors present a serious global health issue with increasing annual incidence and high mortality rates. At present, the available treatment approaches for malignant tumors include surgery, chemotherapy, radiotherapy, targeted therapy, and immunotherapy. Among them, immunotherapy has gradually evolved in recent years. In 2012, 6-year-old Emily Whitehead diagnosed with acute lymphoblastic leukemia (ALL) received CD19-CAR-T cell therapy. Thereafter, the patient achieved complete remission (1). Emily's story elicited an upsurge in tumor immunotherapy and simultaneously opened the prelude to CAR-T cell immunotherapy for tumor treatment.

\section{The Structure and Principle of CAR-T Cells} CAR comprises 4 parts including single-chain variable fragment (scFv), extracellular hinge region, transmembrane region, and intracellular signaling domain (immunoreceptor tyrosine-based activation motif, ITAM) (Figure 1). Notably, extracellular hinge region and $\mathrm{scFv}$ are also referred to as extracellular target binding domain. The extracellular target binding domain recognizing tumor antigens and intracellular signaling domain are recombined in vitro to form recombinant plasmids, then transfected into $\mathrm{T}$ cells of the patient with transfection technology in vitro, hence these $\mathrm{T}$ cells express CARs. Subsequently, these $\mathrm{T}$ cells are expanded and screened in vitro, eventually returning to the body. Since its initial proposal in 1989, CAR has so far developed to the $5^{\text {th }}$ generation (Figure 1).

\section{The Clinical Application of CAR-T Cell Immunotherapy}

CAR-T cell immunotherapy has demonstrated significant curative effects in the treatment of hematologic malignancies. These significant achievements promote its application in the treatment of solid tumors. In contrast with the therapeutic effect of CAR-T cell immunotherapy in hematological malignancies, the general curative effect on solid tumors is insignificant. Therefore, CAR-T cell immunotherapy has great potential in the treatment of solid tumors, providing a novel idea and method for the clinical treatment.

\section{OBSTACLES AND COPING STRATEGIES OF CAR-T CELL IMMUNOTHERAPY IN THE TREATMENT OF SOLID TUMORS}

Significant achievement of CAR-T cell immunotherapy in hematologic malignancies has promoted its application in the treatment of solid tumors. Therefore, several trials currently apply CAR-T against solid tumors, including glioblastoma (2), lung cancer (3), liver cancer (4), gastric cancer (5), renal cancer (6), prostate cancer (7), etc. Nevertheless, based on a metaanalysis on the efficacy of CAR-T in treating solid tumors, CAR$\mathrm{T}$ cell immunotherapy produced a comprehensive response rate of $9 \%(8)$. This is primarily attributed to various obstacle factors, as discussed below.

\section{Lack of Tumor-Specific Antigens (TSAs)}

Lack of TSAs in solid tumors is one of the primary reasons for the poor efficacy of CAR-T cell immunotherapy. In most of the current solid tumors, treatment targets are mostly tumorassociated antigens (TAAs), thus the specificity is not high. This triggers inevitable off-target effects of CAR-T cells in the treatment of solid tumors. Off-target effects sometimes cause severe adverse reactions and even life-threatening outcomes. In one clinical trial, researchers constructed carboxy-anhydrase-IX (CAIX)-targeted CAR-T cells to treat patients diagnosed with advanced renal cell carcinoma (RCC) (9). As a consequence, 4 out of 12 patients were terminated in the experiment because of severe liver damage. This was attributed to CAIX-CAR-T cells damaging the bile duct epithelial cells that express CAIX. 


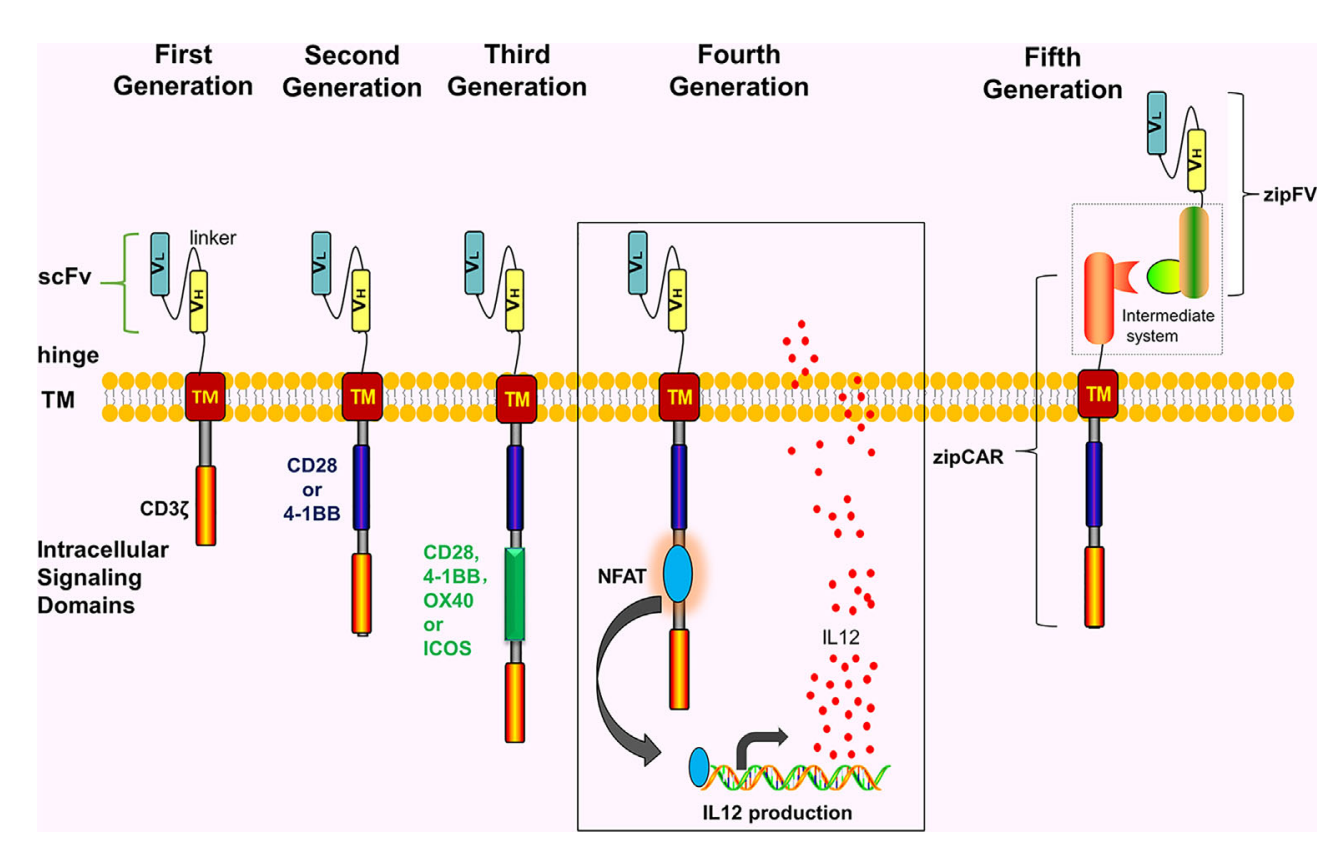

FIGURE 1 | The development of the CARs. The intracellular structure of first-generation CARs only has one signal structure domain (CD3ל) without co-stimulatory molecules. The second-generation CARs add one co-stimulatory molecule to the first-generation CAR, e.g, CD28, 4-1BB, OX40 or ICOS. The third-generation CARs contain 2 costimulatory molecules. The fourth-generation CARs are modified by adding NFAT or suicide genes based on the second-generation CARs or the thirdgeneration CARs. The fifth-generation CARs use a "third-party" intermediate system to separate the antigen-binding domain of CARs from the T cell signaling unit.

Notably, designing CARs for TSAs is an effective approach to solve the off-target effect of CAR-T cell immunotherapy. Nonetheless, these TSAs are extremely rare, therefore, TAAs have to be utilized. Therefore, it is essential to institute measures that enhance the efficiency of CAR-T cells binding to solid tumor cell surface antigens.

\section{Combined CAR-T Cell Immunotherapy}

Combined CAR-T cell immunotherapy refers to the simultaneous or sequential use of 2 or more CAR-T cells for a similar malignant tumor (Figure 2A). This can exhaustively expend TAAs and enhance the ability of CAR-T cells to recognize and bind to the corresponding tumor cells. Feng et al. (10) treated one patient with advanced unresectable/metastatic cholangiocarcinoma (CCA) with a CAR-T cocktail immunotherapy. This therapy involves continuous infusion of epidermal growth factor receptor (EGFR)-CAR-T cells and CD133-CAR-T cells, respectively. As a result, the patient achieved a 13-month partial response (PR). This strategy suggested the feasibility of CAR-T cocktail immunotherapy in the treatment of solid malignancies. The combined use of 2 different CAR-T cells has better efficacy compared to using either CAR-T cell alone (11). However, the pros and cons of this therapy in the prognosis of cancer patients and toxic reactions produced during the treatment still require numerous clinical trials for verification and evaluation. Additionally, reports suggest another combination mode, called dual signaling CAR, i.e., 2 different CARs simultaneously and separately expressed on a similar $\mathrm{T}$ cell surface $(12,13)$ (Figure 2B). One study has revealed that dual- signaling CAR-T cells have better antitumor activity compared to one type of CAR-T cells or 2 types of hybrid CAR-T cells in vivo (12). Also, since the 2 CARs need to be separately constructed, both time and cost remain relatively increased.

\section{Tandem CARs (TanCARs)}

TanCARs adopt the design philosophy of the "OR" gate. Downregulation or mutation of target antigens is often observed in cancer cells, and the loss of target antigens causes cancer cells to escape eventually inducing tumor recurrence (14). Therefore, if TAAs on the tumor cell surface are fully utilized and 2 or more TAAs simultaneously targeted, tumor recurrence caused by target antigen loss can be largely prevented (because the probability of losing both antigens is much lower than the probability of losing one antigen). Based on this principle, Grada et al. (15), designed a bispecific CAR, tanCAR, which connected 2 different scFvs in tandem to a single transgenic receptor (Figure 2C). This series connection allows TanCAR subunits to freely move (15). The tandem CAR-T cell contains 2 different scFvs. CAR is activated when any scFv binds to the tumor cell surface antigen. When both scFvs simultaneously bind to the 2 antigens on the tumor cell surface, the CAR is activated with a synergistic effect which further enhances the activation and tumor-killing ability of CAR-T cells $(15,16)$.

Generally, unlike single antigen-specific CAR or a combination of 2 single antigen-specific CARs, tanCAR effectively prevents the escape of tumor cells and enhances tumor-killing effects with less toxicity $(15,16)$. Moreover, researchers further optimized the CAR by developing a specific type of trivalent CAR-T cells, which 


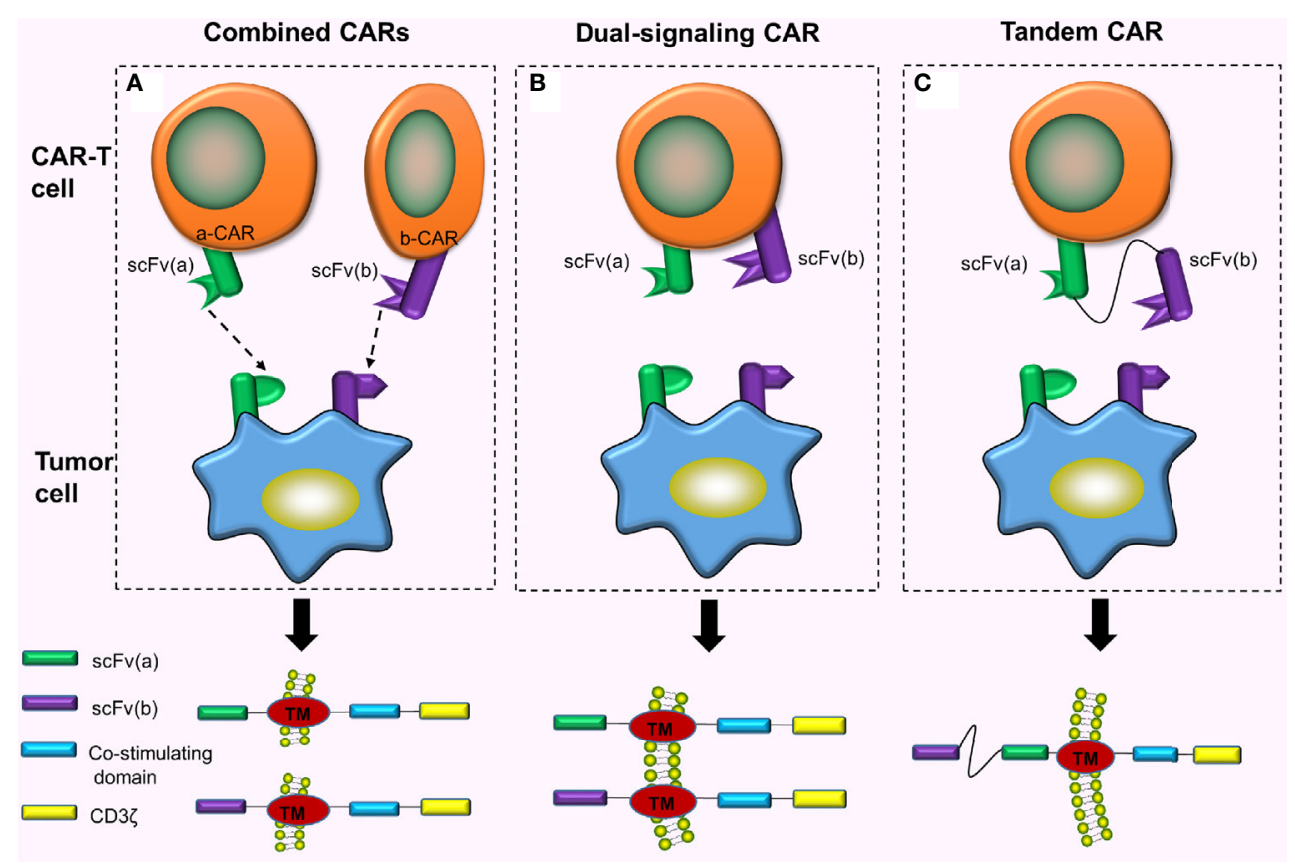

FIGURE 2 | Combined CARs, Dual-singling CAR and Tandem CAR. (A) Simultaneous or sequential use of 2 or more CAR-T cells treating a similar malignant tumor; (B) Dual-signaling CAR refers to the simultaneous and separate expression of 2 different CARs on similar T cell surface; (C) Two different scFvs are connected hand in hand to form tandem CAR on the surface of the T cell.

simultaneously express 3 CAR molecules, targeting HER2, IL13R $\alpha 2$, and EphA2 on the surface of glioblastoma cells. The trivalent CAR-T cells revealed greater anti-glioma activity in mouse models (17). In recent years, tanCARs have been increasingly applied in the research and treatment of solid tumors.

\section{The synNotch AND Gate T Cells (AND Gate CARs)}

CAR-T cells designed for TAAs inevitably produce off-target effects. As such, improving the activity and accuracy of CAR-T cells is an ideal improvement method. Roybal et al. (18) developed a new class of modular receptors, called synNotch receptors. These receptors use the $\mathrm{scFv}$ in binding to the corresponding target antigen $\mathrm{A}$, however, the combination of two does not trigger the $\mathrm{T}$ cells activation, unlike the conventional CARs. Instead, this binding causes the synNotch receptor to split and release the transcriptional activator domain, which enters the nucleus and drives the expression of other BCAR genes. Subsequently, the surface of the T cell express BCARs against the target antigen $\mathrm{B}$. At this time, the newly expressed CARs binds to the target antigen $\mathrm{B}$, eventually activating the T cells (18) (Figure 3).

A few studies reveal that the synNotch AND gate T cells have shown precise recognition and strong tumor-killing ability, which are ineffective for tumor cells expressing a single antigen (can reduce off-target effects), and effectively eliminating tumor cells expressing the corresponding double antigens (19). Research proposes one assumption that combines synNotch receptor with $\operatorname{tanCAR}$ in constructing a new type of CAR-T cells that simultaneously eliminate tumor cells expressing 3 corresponding antigens (20), a hypothesis has recently been tested. Williams et al. (21) effectively constructed this new type of CAR-T cell by transcriptionally linking multiple receptors. These CAR-T cells comprised synNotch receptors targeting hepatocyte growth factor receptor (MET), and tanCARs targeting EGFR and HER2. This new design improved the ability of CAR-T cells to recognize and kill targeted tumor cells (simultaneously expressing [HER2 and/or EGFR] and MET).

\section{Universal CARs}

Notably, the fifth-generation CARs are also called universal CARs. These CARs use a "third party" intermediate system to separate the antigen-binding domain of CAR from the T-cell signaling unit, which theoretically recognizes different antigens without reinventing the T-cell to recognize new antigens (22-25) (Figure 1). In theory, universal CARs minimize tumor cell escape. Universal CARs comprise BBIR (biotin-binding immune receptors) CAR (23) and SUPRA (split universal and programmable) CAR (25). The BBIR CAR system (23) comprises 2 parts, i.e., the BBIR containing dimeric avidin, which binds to the membrane of $\mathrm{T}$ cells; and biotin, which binds to antigen-specific molecules (e.g, scFv, Abs, or tumorspecific ligands). The 2 are combined by non-covalent action to establish the targeted relationship between $\mathrm{T}$ cells and tumor antigens and finally activating $\mathrm{T}$ cells. Also, the SUPRA CAR system (25) comprises 2 parts, i.e., $\mathrm{T}$ cell universal receptor with leucine zipper adaptor (zipCAR) and scFv with leucine zipper adaptor (zipFV) targeting tumor-specific antigens. The targeted 


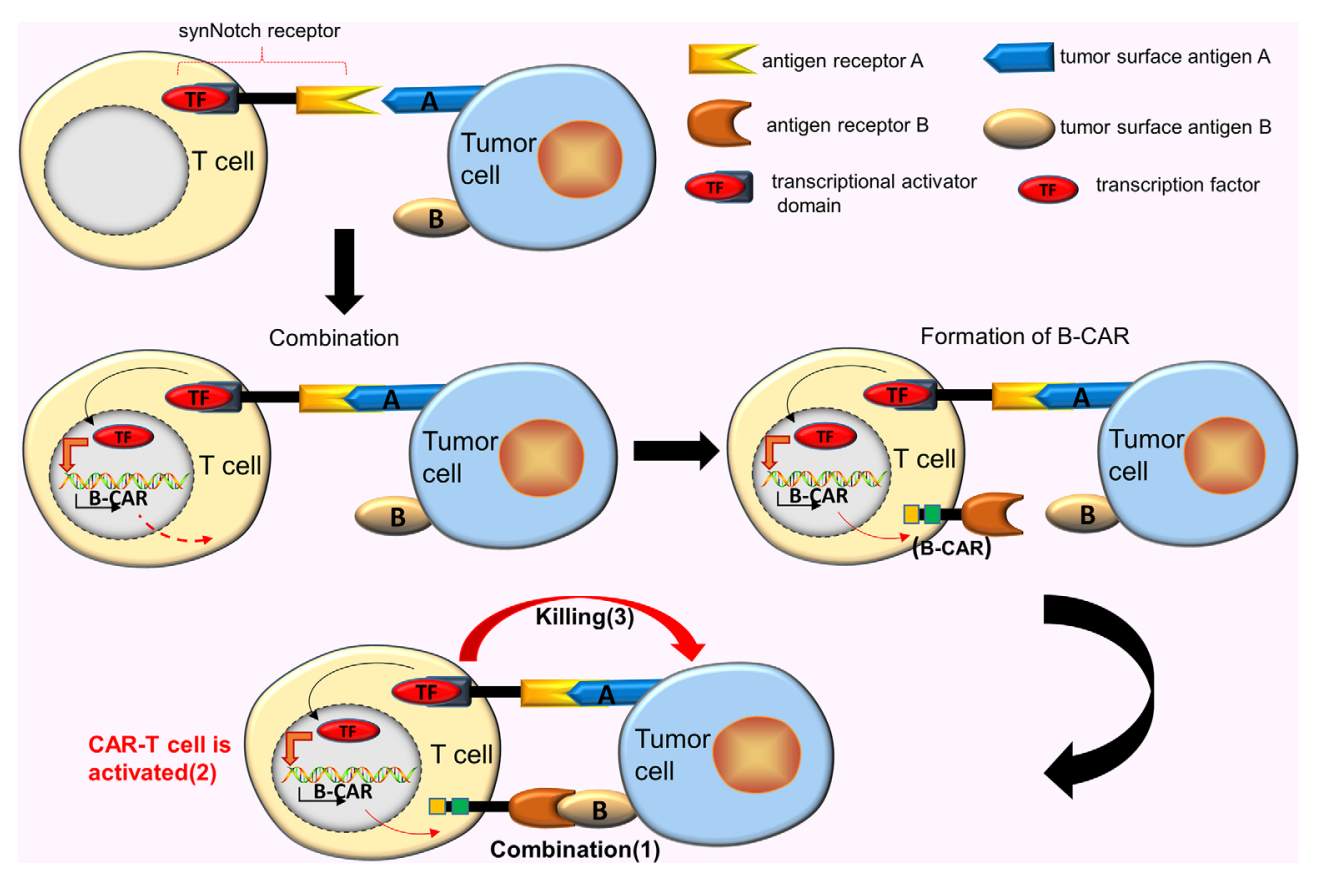

FIGURE 3 | AND gate CAR. SynNotch receptor uses the scFv to bind to the corresponding target antigen A, however, the combination of 2 does not trigger the T cells activation. This binding causes the synNotch receptor to split and release the transcriptional activator domain, which enters the nucleus and drives the expression of another B-CAR gene. Subsequently, the surface of the T cell will express B-CARs. At this time, the newly expressed CARs binds to the target antigen $\mathrm{B}$, activating the $\mathrm{T}$ cells.

relationship between $\mathrm{T}$ cells and tumor antigens is established through a combination of zipCAR and zipFV. And T cells finally are activated. In theory, universal CARs should be the ideal CARs.

\section{Tumor Microenvironment (TME) Inhibits CAR-T Cell Activity}

Tumor microenvironment (TME) refers to the survival environment of tumors. Its components primarily include cancer cells, surrounding immune cells, cancer-associated fibroblasts, stromal tissues, microvasculars, hormones, cytokines and chemokines, etc. (26). TME disrupts and inhibits the activity of CAR-T cells in the treatment of solid tumors, causing the inability of CAR-T cells to effectively recognize and kill tumor cells (27). Therefore, this situation can be enhanced in two ways, i.e., (a) First, based on the characteristics of TME, CAR-T cells that maintain activity and resist immunosuppressive factors in TME can be designed and enhanced; (b) Secondly, remodeling TME to minimize its immunosuppressive effect.

\section{Designing CAR-T Cells Based on the Characteristics of TME}

The glycolytic metabolism of cancer cells causes hypoxia, low $\mathrm{pH}$, and low nutrition in TME, which inhibit the activity of CAR$\mathrm{T}$ cells $(28,29)$. For instance, Juillerat et al. designed hypoxiainducible factors 1-alpha (HIF1 $\alpha$ )-CAR-T cells based on the characteristic of hypoxia in TME. In contrast with normal oxygen concentration, the ability of these CAR-T cells to kill their target cells in a hypoxic environment was significantly enhanced (30). Therefore, CARs can also be designed based on the low $\mathrm{pH}$ and nutritional characteristics of TME.

\section{Improving CAR-T Cells Based on Immunosuppressive Cells or Molecules}

The solid tumor microenvironment is rich in suppressive immune cells, including regulatory $\mathrm{T}$ cells (Tregs), myeloid-derived suppressor cells (MDSCs), tumor-associated neutrophils (TANs), etc. These suppressive immune cells secrete growth factor $\beta$ (TGF- $\beta$ ), IL-10, indoleamine-2, 3-dioxygenase (IDO), etc. which inhibit the activity of CAR-T cells and promote tumor escape $(27,31)$. For example, Kloss et al. (32) designed a type of PSMA-CAR-T cells that simultaneously expressed the dominant-negative TGF- $\beta$ RII (dnTGF- $\beta$ RII). In mouse models of human prostatic cancer, these PSMA-CAR-T cells expressing dnTGF- $\beta$ RII receptors could block TGF- $\beta$ signaling to promote the proliferation and antitumor activity of CAR-T cells.

\section{Remodeling the TME}

To improve TME, researchers designed lipid nanoparticles comprising drug cocktail to remodel TME (i.e., blocking the suppressive immune cells in TME and activating the key antitumor immune cells, which reverses the adverse TME). After maximizing this effect, tumor-specific CAR-T cells are administered (33). Of note, this therapy significantly improves the curative effect of CAR-T cells in solid tumors. 


\section{Homing Barriers of CAR-T Cells}

Homing barriers of CAR-T cells refers to CAR-T cells that cannot efficiently enter solid tumors. CAR-T cells only and effectively kill tumor cells when they migrate to the tumor site, specifically into the interior of the primary tumor and metastasis. There exist 2 major barriers between CAR-T cells and solid tumor cells, including vascular barrier and stromal barrier in solid tumor tissues. Therefore, we can break through these two barriers by taking the measures listed below.

\section{Regional Injection of CAR-T Cells}

Clinically, CAR-T cells are frequently injected into the body via intravenous injection. Nevertheless, due to the barriers between the input CAR-T cells and solid tumor cells, only a small amount of CAR-T cells enter the interior of the solid tumor, most of them remaining in the peripheral circulation. To increase the quantity and concentration of CAR-T cells in the local area, regional injection of CAR-T cells, a safe and effective method can be adopted $(34,35)$. Katz et al. designed CEA-CAR-T cells for patients diagnosed with unresectable CEA+ adenocarcinoma liver metastases (LM), then injected CEA-CAR-T cells into liver metastases with percutaneous hepatic artery infusions (HAIs). Their results revealed that CAR-T cells could be detected in tumors and normal livers, while not or rarely in peripheral blood. Besides, CEA-CAR-T HAIs demonstrated satisfactory safety (no grade 4 or 5 adverse events) and clinical activity (36).

A few clinical or animal studies have shown that regional injection of CAR-T cells in other solid tumors has a better therapeutic effect. For instance, regional intraperitoneal delivery of TAG72-CAR-T cells for the treatment of ovarian cancer (37); and intracranial infusions of interleukin-13 receptor alpha 2 (IL13R $\alpha 2$ )-CAR-T cells for the treatment of recurrent multifocal glioblastoma (38).

\section{Increasing the Penetrability of CAR-T Cells}

A large amount of extracellular matrix (ECM) has been observed around solid tumors and between tumor cells. Notably, the primary component of ECM is heparan sulfate proteoglycans (HSPGs). The heparanase (HPSE) degrade HSPGs. Therefore, researchers designed CAR-T cells that expressed HPSE and enhance their ability to degrade HSPGs, improve the penetration of CAR-T cells, and promote homing as well as infiltration of CAR-T cells in solid tumors (39). Similarly, corresponding CAR-T cells can be designed for other matrix components to enhance homing ability in solid tumors.

\section{Improving the Ability of CAR-T Cells to Aggregate Around Solid Tumors}

Tumor cells secrete chemokines (which act as chemotactic agents for immunosuppressive cells and enhance the immunosuppressive effect of TME), including interleukin-8 (IL-8, CXCL8) (40, 41). Research indicated that CAR-T cells expressing the IL- 8 ligand (CXCR2) could migrate more efficiently to sites where IL- 8 was present and to tumor cells with IL-8 (42). Interestingly, Smith et al. (43) developed a biopolymer device that could place CAR-T cells and place this device on the surface of a solid tumor, thereby increasing the concentration of CAR-T cells around the solid tumor and more effectively killing tumor cells.

\section{CAR-T Cell Differentiation, Increased Depletion, Decreased Proliferation, and Short Duration of Activity}

CAR-T cell differentiation, increased exhaustion, decreased proliferation, and short duration of activity maintenance, weaken the therapeutic effect of CAR-T cells in tumors. Additionally, the metabolic state of CAR-T cells influences the survival and efficacy of CAR-T cells (44). In contrast with hematologic malignancies, the activity maintenance time of CAR-T cells in patients with solid tumors was significantly shortened. These phenomena have been attributed to several reasons including (a) After injecting CAR-T cells into the body, they recognize, bind to, and kill the target cells, causing depletion of CAR-T cells; (b) Suppressive TME; (c) Selecting specific subsets of $\mathrm{T}$ cells to construct CAR-T cells; (d) The design of CAR itself (for example, whether it contains costimulatory molecules and their number); (e) Whether CAR-T cells can be effectively activated in the body; (f) Differentiation type of CAR$T$ cells. Notably, these reasons can be addressed.

For instance, the infusion of CAR-T cells can be intermittent or in increments to guarantee the concentration and total amount of CAR$\mathrm{T}$ cells in the body. The persistence of CAR-T cells is associated with the curative effect. Selecting specific $T$ cell subtypes to construct CAR$\mathrm{T}$ cells (such as $\mathrm{Tn}$ or $\mathrm{Tcm}$ ) or adopting measures to promote the production of these cells enables CAR-T cells to survive longer with a longer anti-tumor effect in the body $(45,46)$. A few researchers used different subtypes of $\mathrm{T}$ cells to construct CAR-T cells (47). Consequently, they discovered that CAR-T cells constructed with either naive $\mathrm{T}$ cells $(\mathrm{Tn})$ or central memory $\mathrm{T}$ cells $(\mathrm{Tcm})$ demonstrated greater proliferation, antitumor activity, and longer duration of activity compared to those constructed with effector memory T cells (Tem). Moreover, CAR-T cells in the body gradually differentiated into shorter effector forms in vivo (i.e., reduced $\mathrm{Tn}$ and $\mathrm{Tcm}$, increased Tem), which was primarily related to the PI3K pathway. Inhibition of PI3K pathway in vivo improved the persistence and efficacy of CAR-T cells (48). Further, by optimizing the manufacturing conditions of CAR-T cells (only changing the initial activation conditions and the medium), more Tcm or stem-like memory $\mathrm{T}$ cells can be differentiated, thereby prolonging the persistence of CAR-T cells and effectively regulating the growth of tumor cells (49).

\section{Immune Checkpoints and CRISPR/Cas9 Technology}

The surface of $\mathrm{T}$ cells comprises numerous molecules with different types and functions. Some molecules provide activation signals, including costimulatory molecules CD28, 4-1BB. Besides, other molecules provide inhibitory signals, called immune checkpoints. When these immune checkpoints bind to the corresponding ligands, inhibitory signals transmitted from tumor cells inhibit the activation of $\mathrm{T}$ cells, resulting in reduced $\mathrm{T}$ cell proliferation and tumor-killing effect (Figure 4A). As such, 


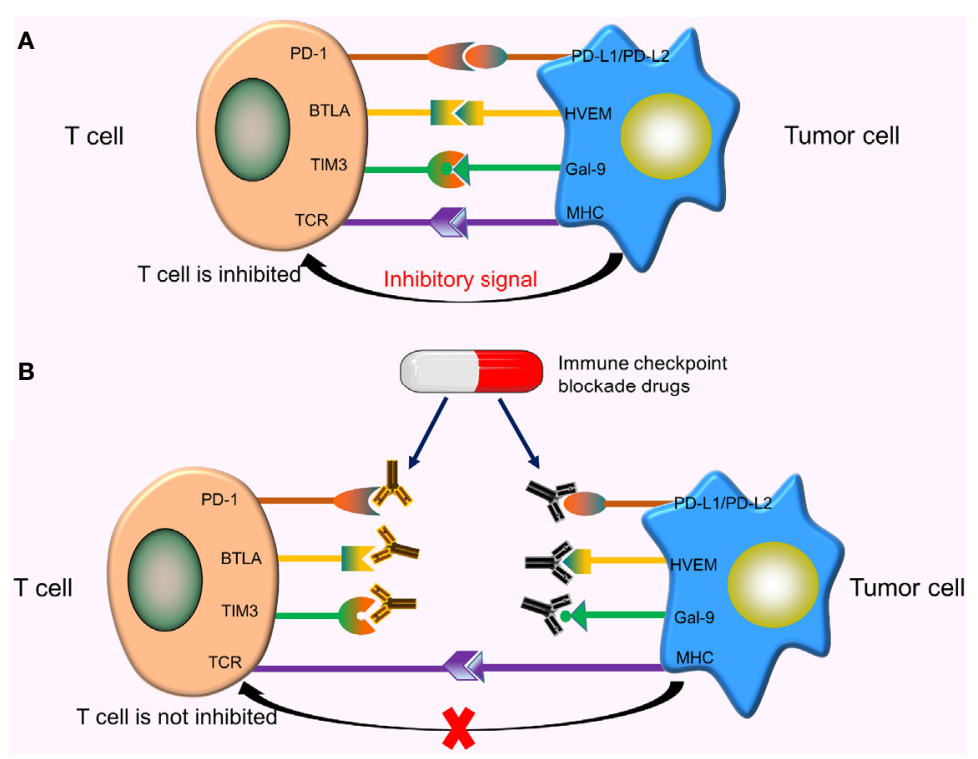

FIGURE 4 | Immune checkpoints and immune checkpoint inhibitors. (A) When these immune checkpoints bind to the corresponding ligands, inhibitory signals transmitted from tumor cells inhibit T cell activation; (B) These immune checkpoint inhibitors specifically bind to the corresponding checkpoints on the T-cell surface or tumor cell surface, thereby blocking the binding of these checkpoints to corresponding ligands, ultimately preventing the transmission of inhibitory signals from tumor cells to $\mathrm{T}$ cells.

tumor cells inhibit $\mathrm{T}$ cell activity by activating immune checkpoints. Currently, the immune checkpoints related to CAR-T cells, including programmed death-1 (PD-1) (50), cytotoxic T lymphocyte-associated protein 4 (CTLA-4) (51), $\mathrm{T}$ cell immunoglobulin mucin domain 3 (TIM3) (52), B and T lymphocyte attenuator (BTLA) (53), etc. The therapeutic effects of CAR-T cells can be enhanced by using immune checkpoint inhibitors, designing special CARS, or using geneediting technology.

\section{Immune Checkpoint Blockade Drugs and CAR-T Cell Immunotherapy}

Common immune checkpoint blockade drugs (immune checkpoint inhibitors) include Nivolumab and Pembrolizumab (PD-1 inhibitors), Atezolizumab and Avelumab (PD-L1 inhibitors), Ipilimumab (a CTLA-4 inhibitor), etc. These inhibitors specifically bind to corresponding checkpoints on the T-cell surface or tumor cell surface, thereby blocking the binding of these checkpoints to the corresponding ligands, and ultimately preventing the transmission of inhibitory signals from tumor cells to T cells (Figure 4B). Studies show that the combined use of CAR-T cells and immune checkpoint inhibitors significantly improve the efficacy of CAR-T cells in solid tumors (54).

\section{Immune Checkpoint and CAR-T Cell Immunotherapy} Immune checkpoints can be used to design special CARs and improve the function of CAR-T cells. Since PD-L1 and PD-L2 are expressed on the surface of tumor cells, CAR-T cells with chimeric PD-1 can be designed. A few researchers adopted this aiming to reverse design CAR for PD-1/PD-L. PD-1 was expressed on the surface of these CAR-T cells, which recognizes the ligands for the
PD-1 receptor expressed in various solid cancers and convert inhibitory signals into activation signals of T cells. Notably, these cells have shown strong antitumor effects in murine models (55). Other researchers designed a type of CAR-T cells. These cells could secrete $\mathrm{scF}$ v that blocked PD-1 to protect CAR-T cells from PD-1/ PD-L1 inhibition. These cells might exhibit better efficacy and higher safety (56).

\section{CRISPR/Cas9 Technology and CAR-T Cell Immunotherapy} Because of low cost, easy operation, and high-efficiency CRISPR/ Cas9 technology has evolved as the most widely used gene-editing technology. Eyquem et al. (57) applied CRISPR/Cas9 technology to construct more powerful CAR-T cells, which delayed the differentiation and exhaustion of effector $\mathrm{T}$ cells and improved the curative effect of CAR-T cells. Moreover, using CRISPR/Cas9 technology knocked out genes associated with immune checkpoints, preventing surface expression of these immune checkpoints on CAR-T cells, thereby improving the persistence and activity of CAR-T cells (58). For instance, $\mathrm{Hu}$ et al. (59) designed CD133-targeted CAR-T cells and knocked out the genes expressing PD-1 with CRISPR/Cas9 technology. Unlike the control group, PD-1-deficient CAR-T cells effectively regulated tumor growth in a mouse model of glioma. The use of CRISPR/Cas9 technology in designing and modifying CAR-T cells will undoubtedly have significant potential in the treatment of solid tumors in the future.

\section{The Trogocytosis of CAR-T Cells}

Mechanisms and reasons for tumor immune escape are complex puzzling scientists for a long time. A new study offers a 
subversive insight, i.e., the key factor in tumor immune escape in CAR-T turns out to be the CAR-T cells. Hamieh et al. (60) simulated the recurrence process of CD19-CAR-T cells in the treatment of ALL in mouse models with ALL. This study uncovers the mechanism of tumor immune escape in CAR-T cell immunotherapy (60), i.e., (a) The CAR-T cells accidentally ingest the surface antigens of tumor cells via trogocytosis, then transfer these antigens to the surface of CAR-T cells, causing them to attack each other, ultimately causing the depletion of the CAR-T cells; (b) When CD19 expression on tumor cell surface is reduced to a certain extent (caused by trogocytosis), the CAR-T cells release inhibitory molecules which prevent cannibalism, and enables tumor cells with low CD19 expression to escape from the chase of the CAR-T cells (Figure 5).

Researchers discovered that CAR-CD19-CD28-T cells and CAR-CD19-4-1BB-T cells exhibited different sensitivity to the density of tumor cell surface antigens. Thus, they combined these 2 types of CAR-T cells (with different costimulatory molecules) to counteract the effect of trogocytosis and significantly enhanced the therapeutic effect (60). This mechanism of immune escape might be prevalent in CAR-T, thus trogocytosis of CAR-T cells can be counteracted via a combined use of different types of CAR-T cells.

\section{Tumor Antigen Heterogeneity}

Tumor heterogeneity refers to the formation of cell subsets with different biological characteristics in the growth process of tumors after multiple division and proliferation. It is one of the characteristics of malignant tumors and a vital factor in cancer treatment failure, cancer recurrence and drug resistance (61). Also, malignant tumor antigens are highly heterogeneous.

\section{Tumor Antigen Heterogeneity Promotes Tumor Immune Escape}

For the same patient, even if the tumor at the same site or even the tumor cells on the same tumor may not express the same tumor antigens (62). Besides, the number or types of tumor antigens are different before and after tumor treatment, as well as in the original and recurrent lesions $(14,31,63)$. Conventional CAR-T cells only target one tumor surface antigen, thereby allowing tumor cells that do not express or under-express that antigen to escape, ultimately causing tumor recurrence. This phenomenon is similar to that when patients take broad-spectrum antibiotics for a long period, sensitive bacteria are inhibited, while insensitive bacteria or fungus maximize the opportunity to multiply and grow, eventually causing superinfection. Therefore, tumor antigen heterogeneity increases the difficulty of solid tumor treatment and the possibility of tumor recurrence.

\section{How to Deal With Tumor Antigen Heterogeneity in CAR-T Cell Immunotherapy}

By solving the problem of tumor antigen heterogeneity, the recurrence rate of tumors can be theoretically reduced. The following measures can be adopted to deal with tumor antigen heterogeneity: (a) Identifying and using TSAs as targets for CAR$\mathrm{T}$ cells which is also an ideal method; (b) Using combined CAR$\mathrm{T}$ cell immunotherapy, tandem CARs, or AND gate CARs to cover as much TAAs of tumor cell surface as possible to counteract tumor antigen heterogeneity; (c) Increasing the density of target antigens. For instance, one study revealed that Bryostatin1 (one drug) could up-regulate the expression of CD22 in leukemia cell lines and lymphoma cell lines, thus improving the function and persistence of CAR-T cells in the body (64); (d)

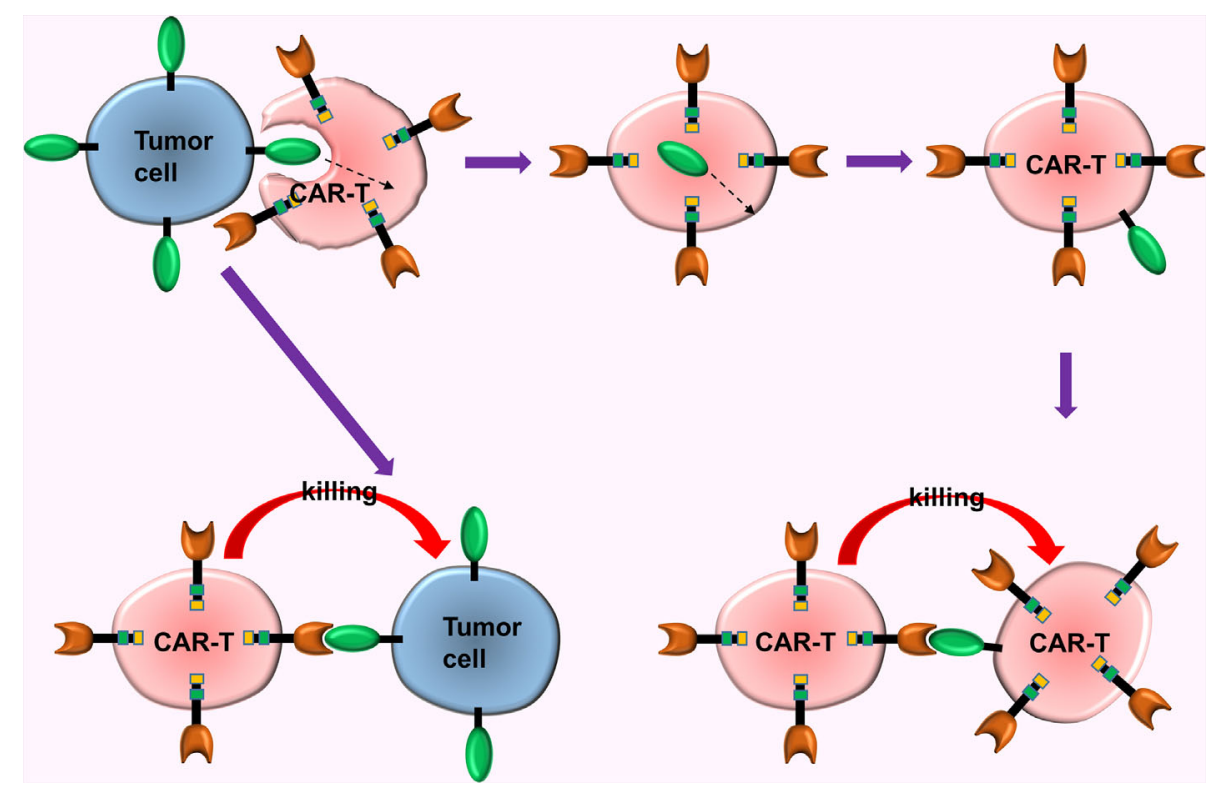

FIGURE 5 | The trogocytosis of CAR-T cells. The CAR-T cells accidentally ingested surface antigens of tumor cells via trogocytosis, and transferred these antigens to the surface of CAR-T cells, causing them to attack each other, ultimately causing the depletion of the CAR-T cells. 
Increasing the affinity of CAR-T cells. For example, Drent et al. (65) discovered that the co-stimulator CD28 and 4-1BB were simultaneously used to build a CAR, increasing the affinity of CAR-T cells. This CAR was highly safe significantly improving its anti-tumor performance in vivo, and retaining its ability to recognize target antigen density; (e) Optimizing the structure of CAR and reducing the threshold of CAR reaction. Majzner et al. (66) added additional ITAM to CAR to enhance signal strength, hence enabling the CAR-T cells to recognize low-antigen-density tumor cells. Also, the CAR with the addition of a CD28-hingetransmembrane region reduced the threshold of CAR response, thereby enhancing the activity of CAR-T cells against lowantigen-density tumor cells; (f) Designing the CARs that can recruit other innate immune cells to kill tumor cells e.g., the fourth-generation CARs (67); (g) The universal CARs with characteristics enabling them to target different tumor antigens and effectively fight against tumor antigen heterogeneity.

\section{Reducing Toxicity by Controlling the Activity of CAR-T Cells}

CAR-T cells inevitably produce toxic effects in the treatment of cancer, including CRS, CRES, off-target effects, etc. If the activity of CAR-T cells in the body can be regulated in a timely and appropriate manner to reduce toxicity, the CAR-T will be safer and more widely used. To minimize these toxic reactions, optimizing the production method of CAR-T cells and the structure of CARs is necessary.

\section{Transient mRNA-Mediated CAR Expression}

Transient mRNA-mediated expression of CAR usually adopts electroporation to transfer the mRNA encoding the target genes into the cytoplasm of $\mathrm{T}$ cells (the mRNA does not enter into the nucleus, thus insertion mutations are extremely rare). These mRNAs encode the corresponding proteins eventually expressing corresponding CARs on the surface of T cells. The instability and short survival time of the mRNA can lead to transient expression of the encoded proteins. Therefore, CARs expressed on the surface of T cells are transient, thus improving the safety of CAR-T cells. One study reported that more than $80 \%$ of CAR-T cells transfected with electroporation survived, while $94 \%$ of these cells were expressed CARs (68).

mRNA electroporation is presently a relatively safe and costeffective method for $\mathrm{T}$ cell gene transduction, and it is more and more widely used in CAR-T cell immunotherapy. Due to the transient nature of the CAR-T cells produced by this method, several rounds of CAR-T cell infusion are required during treatment. Nevertheless, repeated injections of CAR-T cells might induce anaphylaxis (69).

\section{Suicide Genes}

The addition of suicide genes to CAR-T cells irreversibly induces apoptosis in the CAR-T cells that cause the toxic reaction, ensuring the safety of CAR-T cell immunotherapy (70). There exist 3 commonly used suicide genes, including (a) Herpes simplex virus thymidine kinase (HSV-tk), which phosphorylate ganciclovir (GCV), transforming it into toxic GCV-triphosphate compound, affecting DNA synthesis and ultimately inducing CAR-T cell death
(71). Since interfering DNA synthesis to induce T cell death is a gradual process, HSV-TK takes a longer time to eliminate the CART cells (72); (b) The caspase 9 (iCasp9) suicide genes, which can be induced into dimerization by AP1903 (one chemical inducer of dimerization, CID), rapidly inducing CAR-T cells apoptosis and preferentially killing activated CAR-T cells with strong transcriptional activity (73). Notably, this further enhances the safety of CAR-T cell immunotherapy; (c) CD20 and truncated epidermal growth factor receptor (EGFRt), i.e., CAR-T cells coexpressing CD20 or EGFRt are constructed. When severe toxicity occurs during treatment, rituximab (a CD20-targeted drug) and cetuximab (an EGFRt-targeted drug) are injected, respectively, inducing antibody-dependent cell-mediated cytotoxicity (ADCC) effect to eliminate these CAR-T cells $(74,75)$. The suicide genes are installed in CAR-T cells to eliminate CAR-T cells causing toxic reactions. Despite regulating toxic reactions, they also reduce the number of CAR-T cells and affect the efficacy.

\section{On-Switch CAR}

To regulate the activity of CAR-T cells in the body and reduce toxicity, researchers developed an on-switch CAR system (76, 77). This new type of on-switch CAR-T cell is initially in a closed state. Only after the use of specially designed drugs, these cells are activated, then identify, bind and kill the targeted tumor cells (Figure 6A). When the drug is not present, CAR-T cells return to their off state, e.g., the on-switch CAR designed by Wu et al. (76), whose the intracellular signal domain was divided into two separate units (i.e., the co-stimulating domain and $\mathrm{CD} 3 \zeta$ ). The co-stimulating domain and $\mathrm{CD} 3 \zeta$ can only be reassembled in the presence of a heterodimerizing small molecule (rapamycin analog AP21967). At this time, the CAR-T cells are activated. By adjusting the dosage of AP21967, time, location, and dose of CAR-T cell activity in vivo can be precisely regulated, thereby reducing the toxicity.

\section{Inhibitory CAR (iCAR)-T Cell}

Fedorov et al. (78) designed a special CAR-T cell called iCAR-T cell that reduces off-target effects. This cell surface simultaneously expresses traditional CARs and iCARs or traditional TCRs and iCARs (Figure 6B). The conventional CARs or TCRs target tumor cells, and the iCARs target normal cells. The iCAR comprises a surface antigen recognition area (which recognizes the normal cells) and an acute inhibition signal area (based on CTLA-4 or PD-1). When the iCAR-T cell binds to the corresponding tumor cell, it will be activated and then kills the tumor cell. However, when the iCAR-T cell combines with the normal cell, iCAR generates inhibitory signals preventing traditional CARs or TCRs on the surface of an iCAR-T cell from functioning, hence the normal cell remains undamaged. Moreover, this effect is reversible. When these cells meet and bind to the targeted tumor cells again, they can still be activated and kill the tumor cells. These characteristics enable iCAR-T cells to distinguish tumor cells from normal cells (78). Although research on iCAR is extremely rare, its design theory is feasible and innovative. One of the major difficulties is the need to identify tissue-specific antigens for iCAR that are missing or down-regulated in tumors but highly expressed in normal tissues. 

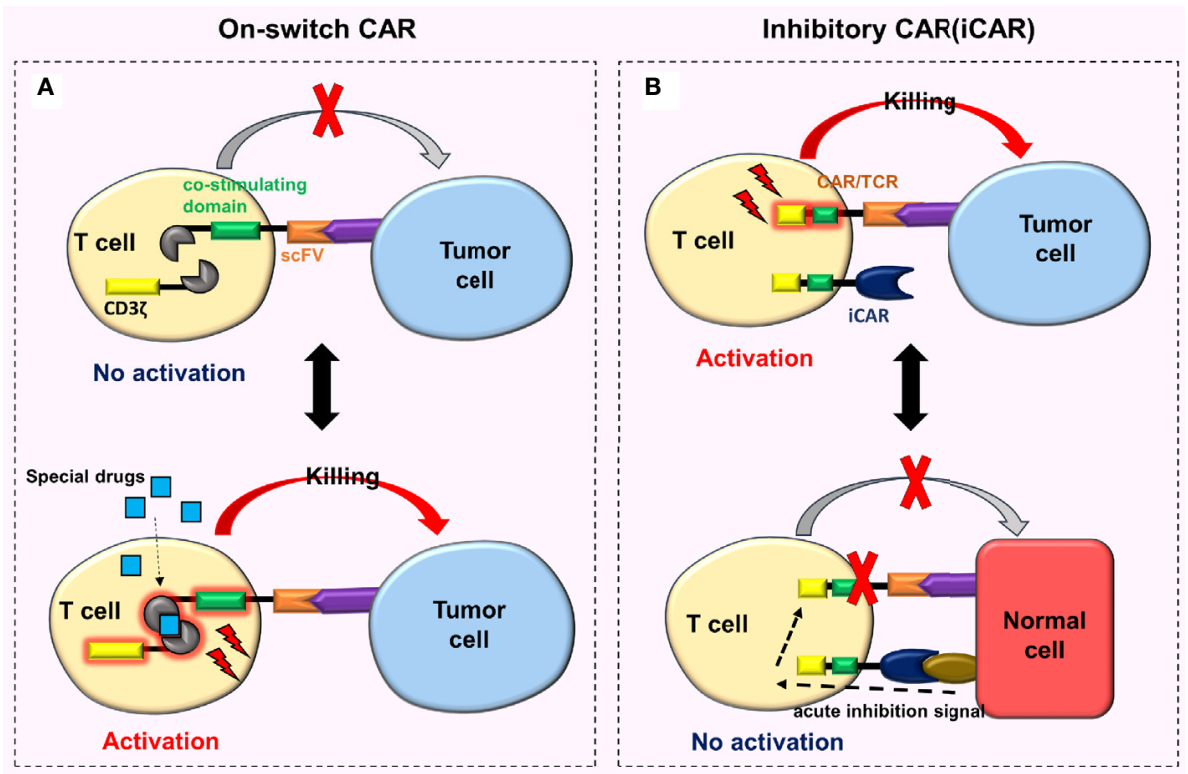

FIGURE 6 | On-switch CAR and iCAR. (A) The on-switch CAR-T cells are initially turned off, activated only with specially designed drugs, then recognize, bind to, and kill the targeted tumor cells. When the drugs are not present, the CAR-T cells return to their off state; (B) When iCAR-T cells bind to the corresponding tumor cells, iCAR-T cells are activated and kill the tumor cells. Nonetheless, when iCAR-T cells combine with normal cells, the iCARs produce inhibitory signals which prevent traditional CARs or TCRs from functioning, thus the normal cells remain undamaged.

\section{Other Improved Methods of CAR-T Cell Immunotherapy}

In recent years, CAR-T has rapidly developed. In addition to the above-mentioned coping strategies in the treatment of solid tumors, many other improved methods have been reported, for example, CAR-T cells combined with tumor vaccine treatment (79); CAR-T cells combined with radiotherapy or chemotherapy (80, 81); CAR-T cells combined with oncolytic viruses (82); CAR-T cells combined with photothermal therapy (83); CAR-T cells combined with nanotechnology (84), etc.

\section{ECONOMIC RESTRICTION}

CAR-T cell immunotherapy is extremely expensive. The currently available 2 CAR-T cell immunotherapy in the market, i.e., Kymriah (Novartis) and Yescarta (Kite Pharma), have an average expected cost of $\$ 510,963$ and $\$ 402,647$ per patient, respectively (85) (conservative estimate). The high cost significantly limits the development and clinical application of CAR-T cell immunotherapy. Therefore, reducing its research and development expenses and clinical use costs is a major problem at the moment

At present, the manufacturing process of CAR-T cells is still in the "manual stage", where most operations need to be manually completed, therefore, the output is limited by expensive cost. At the $3^{\text {rd }}$ Global CAR-TCR Summit in 2017, experts believed that accelerating automated production is vital to reducing the cost of CAR-T cell immunotherapy. As such, there is an urgent need to upgrade the CAR-T cell production process. Notably, automatic, fully enclosed, and cGMP (current good manufacture practices)-compliant CAR-T cell preparation is a major trend in the future $(86,87)$. Meanwhile, other approaches to reducing the cost of CAR-T cells have been reported. For example, electroporation technology significantly shortens the production time of CAR-T cells, reducing the production cost, with a high curative effect and few adverse reactions (88). Shortening the time of in vitro culture (can be shortened to 3 days) prevents the differentiation of CAR-T cells during amplification (i.e., the reduction of Tscm memory stem cells is prevented), which is simple, cost-effective, and most importantly, it significantly improves the proliferation ability and tumor-killing effect of CAR-T cells (89). Additionally, TSAs on the surface of tumor cells are the most ideal targets for CAR-T cells, however, identifying new TSAs remains difficult. Nextgeneration sequencing technologies are usually used to screen neoantigens (90), nevertheless, they have a high screening cost. As such, known TAAs can be used for in-depth research.

\section{PROSPECT AND SUMMARY OF CAR-T CELL IMMUNOTHERAPY}

As an emerging tumor immunotherapy, CAR-T cell immunotherapy has a remarkable development and significant potential in the treatment of tumors. Besides, it has demonstrated notable future opportunities in the mainstream cancer treatment of solid tumors. CAR-T cell immunotherapy still has numerous obstacles in the treatment of solid tumors, including the lack of specific targets, 
TME inhibition, CAR-T cells homing obstacle, the trogocytosis of CAR-T cells, etc. Nonetheless, with the continuous breakthrough of these problems, CAR-T cell immunotherapy will exert better curative effects in the treatment of solid tumors, and might even be one of the primary treatment options in the future.

\section{AUTHOR CONTRIBUTIONS}

LM: Writing-Original draft preparation, Investigation, and figure preparation. ZZ: Investigation and figure preparation. ZR: Investigation. FT: Investigation. YL: Conceptualization,

\section{REFERENCES}

1. Grupp SA, Kalos M, Barrett D, Aplenc R, Porter DL, Rheingold SR, et al. Chimeric Antigen Receptor-Modified T Cells for Acute Lymphoid Leukemia. New Engl J Med (2013) 368(16):1509-18. doi: 10.1056/NEJMoa1215134

2. Ahmed N, Brawley V, Hegde M, Bielamowicz K, Kalra M, Landi D, et al. Her2-Specific Chimeric Antigen Receptor-Modified Virus-Specific T Cells for Progressive Glioblastoma: A Phase 1 Dose-Escalation Trial. JAMA Oncol (2017) 3(8):1094-101. doi: 10.1001/jamaoncol.2017.0184

3. Hu Z, Zheng X, Jiao D, Zhou Y, Sun R, Wang B, et al. Lunx-CAR T Cells as a Targeted Therapy for Non-Small Cell Lung Cancer. Mol Ther Oncolytics (2020) 17:361-70. doi: 10.1016/j.omto.2020.04.008

4. Batra SA, Rathi P, Guo L, Courtney AN, Fleurence J, Balzeau J, et al. Glypican3-Specific Car T Cells Coexpressing IL15 and IL21 Have Superior Expansion and Antitumor Activity Against Hepatocellular Carcinoma. Cancer Immunol Res (2020) 8(3):309-20. doi: 10.1158/2326-6066.Cir-19-0293

5. Jiang H, Shi Z, Wang P, Wang C, Yang L, Du G, et al. Claudin18.2Specific Chimeric Antigen Receptor Engineered T Cells for the Treatment of Gastric Cancer. J Natl Cancer Inst (2019) 111(4):409-18. doi: 10.1093/ jnci/djy134

6. Li H, Ding J, Lu M, Liu H, Miao Y, Li L, et al. CAIX-Specific CAR-T Cells and Sunitinib Show Synergistic Effects Against Metastatic Renal Cancer Models. J Immunother (Hagerstown Md: 1997) (2020) 43(1):16-28. doi: 10.1097/ cji.0000000000000301

7. Gorchakov AA, Kulemzin SV, Kochneva GV, Taranin AV. Challenges and Prospects of Chimeric Antigen Receptor T-Cell Therapy for Metastatic Prostate Cancer. Eur Urol (2020) 77(3):299-308. doi: 10.1016/j.eururo. 2019.08.014

8. Hou B, Tang Y, Li W, Zeng Q, Chang D. Efficiency of CAR-T Therapy for Treatment of Solid Tumor in Clinical Trials: A Meta-Analysis. Dis Markers (2019) 2019:3425291. doi: 10.1155/2019/3425291

9. Lamers CH, Sleijfer S, van Steenbergen S, van Elzakker P, van Krimpen B, Groot C, et al. Treatment of Metastatic Renal Cell Carcinoma With CAIX CAR-Engineered T Cells: Clinical Evaluation and Management of on-Target Toxicity. Mol Ther (2013) 21(4):904-12. doi: 10.1038/mt.2013.17

10. Feng KC, Guo YL, Liu Y, Dai HR, Wang Y, Lv HY, et al. Cocktail Treatment With EGFR-specific and CD133-specific Chimeric Antigen ReceptorModified T Cells in a Patient With Advanced Cholangiocarcinoma. J Hematol Oncol (2017) 10(1):4. doi: 10.1186/s13045-016-0378-7

11. Wei X, Lai Y, Li J, Qin L, Xu Y, Zhao R, et al. PSCA and MUC1 in non-SmallCell Lung Cancer as Targets of Chimeric Antigen Receptor T Cells. Oncoimmunology (2017) 6(3):e1284722. doi: 10.1080/2162402x.2017.1284722

12. Ruella M, Barrett DM, Kenderian SS, Shestova O, Hofmann TJ, Perazzelli J, et al. Dual CD19 and CD123 Targeting Prevents Antigen-Loss Relapses After CD19-directed Immunotherapies. J Clin Invest (2016) 126(10):3814-26. doi: $10.1172 /$ jci87366

13. Zhao Z, Xiao X, Saw PE, Wu W, Huang H, Chen J, et al. Chimeric Antigen Receptor T Cells in Solid Tumors: A War Against the Tumor Microenvironment. Sci China Life Sci (2020) 63(2):180-205. doi: 10.1007/ s11427-019-9665-8

14. Sotillo E, Barrett DM, Black KL, Bagashev A, Oldridge D, Wu G, et al. Convergence of Acquired Mutations and Alternative Splicing of CD19
Methodology, Supervision. All authors contributed to the article and approved the submitted version.

\section{FUNDING}

This work was supported by Special Research Project of Lanzhou University Serving the Economic and Social Development of Gansu Province (054000282), Lanzhou Talent Innovation and Entrepreneurship Project (2020-RC-38), and Fundamental Research Funds for the Central Universities (lzujbky2020-kb14).

Enables Resistance to CART-19 Immunotherapy. Cancer Discovery (2015) 5 (12):1282-95. doi: 10.1158/2159-8290.Cd-15-1020

15. Grada Z, Hegde M, Byrd T, Shaffer DR, Ghazi A, Brawley VS, et al. Tancar: A Novel Bispecific Chimeric Antigen Receptor for Cancer Immunotherapy. $\mathrm{Mol}$ Ther Nucleic Acids (2013) 2(7):e105. doi: 10.1038/mtna.2013.32

16. Schneider D, Xiong Y, Wu D, Nölle V, Schmitz S, Haso W, et al. A Tandem CD19/CD20 CAR Lentiviral Vector Drives on-Target and Off-Target Antigen Modulation in Leukemia Cell Lines. J Immunother Cancer (2017) 5:42. doi: 10.1186/s40425-017-0246-1

17. Bielamowicz K, Fousek K, Byrd TT, Samaha H, Mukherjee M, Aware N, et al. Trivalent CAR T Cells Overcome Interpatient Antigenic Variability in Glioblastoma. Neuro-oncology (2018) 20(4):506-18. doi: 10.1093/neuonc/nox182

18. Roybal KT, Rupp LJ, Morsut L, Walker WJ, McNally KA, Park JS, et al. Precision Tumor Recognition by T Cells With Combinatorial AntigenSensing Circuits. Cell (2016) 164(4):770-9. doi: 10.1016/j.cell.2016.01.011

19. Srivastava S, Salter AI, Liggitt D, Yechan-Gunja S, Sarvothama M, Cooper K, et al. Logic-Gated ROR1 Chimeric Antigen Receptor Expression Rescues T Cell-Mediated Toxicity to Normal Tissues and Enables Selective Tumor Targeting. Cancer Cell (2019) 35(3):489-503.e8. doi: 10.1016/j.ccell.2019. 02.003

20. Sabahi M, Jabbari P, Alizadeh Haghighi M, Soltani S, Soudi S, Rahmani F, et al. Proposing a Tandem AND-gate Car T Cell Targeting Glioblastoma Multiforme. Med Hypotheses (2020) 137:109559. doi: 10.1016/j.mehy.2020. 109559

21. Williams JZ, Allen GM, Shah D, Sterin IS, Kim KH, Garcia VP, et al. Precise T Cell Recognition Programs Designed by Transcriptionally Linking Multiple Receptors. Sci (New York NY) (2020) 370(6520):1099-104. doi: 10.1126/ science.abc6270

22. Zhao J, Lin Q, Song Y, Liu D. Universal CARs, Universal T Cells, and Universal CAR T Cells. J Hematol Oncol (2018) 11(1):132. doi: 10.1186/ s13045-018-0677-2

23. Urbanska K, Lanitis E, Poussin M, Lynn RC, Gavin BP, Kelderman S, et al. A Universal Strategy for Adoptive Immunotherapy of Cancer Through Use of a Novel T-cell Antigen Receptor. Cancer Res (2012) 72(7):1844-52. doi: 10.1158/0008-5472.Can-11-3890

24. Ma JS, Kim JY, Kazane SA, Choi SH, Yun HY, Kim MS, et al. Versatile Strategy for Controlling the Specificity and Activity of Engineered T Cells. Proc Natl Acad Sci USA (2016) 113(4):E450-8. doi: 10.1073/pnas.1524193113

25. Cho JH, Collins JJ, Wong WW. Universal Chimeric Antigen Receptors for Multiplexed and Logical Control of T Cell Responses. Cell (2018) 173 (6):1426-38. doi: 10.1016/j.cell.2018.03.038

26. Quail DF, Joyce JA. Microenvironmental Regulation of Tumor Progression and Metastasis. Nat Med (2013) 19(11):1423-37. doi: 10.1038/nm.3394

27. Zhang E, Gu J, Xu H. Prospects for Chimeric Antigen Receptor-Modified T Cell Therapy for Solid Tumors. Mol Cancer (2018) 17(1):7. doi: 10.1186/ s12943-018-0759-3

28. Renner K, Singer K, Koehl GE, Geissler EK, Peter K, Siska PJ, et al. Metabolic Hallmarks of Tumor and Immune Cells in the Tumor Microenvironment. Front Immunol (2017) 8:248. doi: 10.3389/fimmu.2017.00248

29. Berahovich R, Liu X, Zhou H, Tsadik E, Xu S, Golubovskaya V, et al. Hypoxia Selectively Impairs CAR-T Cells In Vitro. Cancers (2019) 11(5):602. doi: $10.3390 /$ cancers 11050602 
30. Juillerat A, Marechal A, Filhol JM, Valogne Y, Valton J, Duclert A, et al. An Oxygen Sensitive Self-Decision Making Engineered CAR T-Cell. Sci Rep (2017) 7:39833. doi: 10.1038/srep39833

31. Newick K, O’Brien S, Moon E, Albelda SM. Car T Cell Therapy for Solid Tumors. Annu Rev Med (2017) 68:139-52. doi: 10.1146/annurev-med062315-120245

32. Kloss CC, Lee J, Zhang A, Chen F, Melenhorst JJ, Lacey SF, et al. DominantNegative Tgf- $\beta$ Receptor Enhances PSMA-Targeted Human Car T Cell Proliferation and Augments Prostate Cancer Eradication. Mol Ther (2018) 26(7):1855-66. doi: 10.1016/j.ymthe.2018.05.003

33. Zhang F, Stephan SB, Ene CI, Smith TT, Holland EC, Stephan MT. Nanoparticles That Reshape the Tumor Milieu Create a Therapeutic Window for Effective T-Cell Therapy in Solid Malignancies. Cancer Res (2018) 78(13):3718-30. doi: 10.1158/0008-5472.Can-18-0306

34. Brown CE, Aguilar B, Starr R, Yang X, Chang WC, Weng L, et al. Optimization of IL13R $\alpha 2$-Targeted Chimeric Antigen Receptor T Cells for Improved Anti-Tumor Efficacy Against Glioblastoma. Mol Ther (2018) 26 (1):31-44. doi: 10.1016/.j.ymthe.2017.10.002

35. Theruvath J, Sotillo E, Mount CW, Graef CM, Delaidelli A, Heitzeneder S, et al. Locoregionally Administered B7-H3-targeted Car T Cells for Treatment of Atypical Teratoid/Rhabdoid Tumors. Nat Med (2020) 26(5):712-9. doi: 10.1038/s41591-020-0821-8

36. Katz SC, Burga RA, McCormack E, Wang LJ, Mooring W, Point GR, et al. Phase I Hepatic Immunotherapy for Metastases Study of Intra-Arterial Chimeric Antigen Receptor-Modified T-cell Therapy for CEA+ Liver Metastases. Clin Cancer Res (2015) 21(14):3149-59. doi: 10.1158/1078-0432.Ccr-14-1421

37. Murad JP, Kozlowska AK, Lee HJ, Ramamurthy M, Chang WC, Yazaki P, et al. Effective Targeting of TAG72(+) Peritoneal Ovarian Tumors Via Regional Delivery of CAR-Engineered T Cells. Front Immunol (2018) 9:2268. doi: 10.3389/fimmu.2018.02268

38. Brown CE, Alizadeh D, Starr R, Weng L, Wagner JR, Naranjo A, et al. Regression of Glioblastoma After Chimeric Antigen Receptor T-Cell Therapy. New Engl J Med (2016) 375(26):2561-9. doi: 10.1056/NEJMoa1610497

39. Caruana I, Savoldo B, Hoyos V, Weber G, Liu H, Kim ES, et al. Heparanase Promotes Tumor Infiltration and Antitumor Activity of CAR-Redirected T Lymphocytes. Nat Med (2015) 21(5):524-9. doi: 10.1038/nm.3833

40. Yan $P$, Zhu H, Yin L, Wang L, Xie P, Ye J, et al. Integrin $\alpha v \beta 6$ Promotes Lung Cancer Proliferation and Metastasis Through Upregulation of IL-8-Mediated Mapk/Erk Signaling. Trans Oncol (2018) 11(3):619-27. doi: 10.1016/j.tranon. 2018.02.013

41. Jin L, Tao H, Karachi A, Long Y, Hou AY, Na M, et al. CXCR1- or CXCR2modified Car T Cells Co-Opt IL-8 for Maximal Antitumor Efficacy in Solid Tumors. Nat Commun (2019) 10(1):4016. doi: 10.1038/s41467-019-11869-4

42. Whilding LM, Halim L, Draper B, Parente-Pereira AC, Zabinski T, Davies $\mathrm{DM}$, et al. Car T-Cells Targeting the Integrin $\alpha \mathrm{v} \beta 6$ and Co-Expressing the Chemokine Receptor CXCR2 Demonstrate Enhanced Homing and Efficacy Against Several Solid Malignancies. Cancers (2019) 11(5):674. doi: 10.3390/ cancers 11050674

43. Smith TT, Moffett HF, Stephan SB, Opel CF, Dumigan AG, Jiang X, et al. Biopolymers Codelivering Engineered T Cells and STING Agonists can Eliminate Heterogeneous Tumors. J Clin Invest (2017) 127(6):2176-91. doi: $10.1172 /$ jci87624

44. Kawalekar OU, O’Connor RS, Fraietta JA, Guo L, McGettigan SE, Posey ADJr., et al. Distinct Signaling of Coreceptors Regulates Specific Metabolism Pathways and Impacts Memory Development in CAR T Cells. Immunity (2016) 44(2):380-90. doi: 10.1016/j.immuni.2016.01.021

45. Toews K, Grunewald L, Schwiebert S, Klaus A, Winkler A, Ali S, et al. Central Memory Phenotype Drives Success of Checkpoint Inhibition in Combination With CAR T Cells. Mol Carcinog (2020) 59(7):724-35. doi: $10.1002 / \mathrm{mc} .23202$

46. Blaeschke F, Stenger D, Kaeuferle T, Willier S, Lotfi R, Kaiser AD, et al. Induction of a Central Memory and Stem Cell Memory Phenotype in Functionally Active CD4(+) and CD8(+) Car T Cells Produced in an Automated Good Manufacturing Practice System for the Treatment of CD19(+) Acute Lymphoblastic Leukemia. Cancer Immunol Immunother: CII (2018) 67(7):1053-66. doi: 10.1007/s00262-018-2155-7

47. Sommermeyer D, Hudecek M, Kosasih PL, Gogishvili T, Maloney DG, Turtle CJ, et al. Chimeric Antigen Receptor-Modified T Cells Derived From Defined
CD8+ and CD4+ Subsets Confer Superior Antitumor Reactivity. vivo Leuk (2016) 30(2):492-500. doi: 10.1038/leu.2015.247

48. Zheng W, O'Hear CE, Alli R, Basham JH, Abdelsamed HA, Palmer LE, et al. PI3K Orchestration of the In Vivo Persistence of Chimeric Antigen ReceptorModified T Cells. Leukemia (2018) 32(5):1157-67. doi: 10.1038/s41375-0170008-6

49. Gargett T, Truong N, Ebert LM, Yu W, Brown MP. Optimization of Manufacturing Conditions for Chimeric Antigen Receptor T Cells to Favor Cells With a Central Memory Phenotype. Cytotherapy (2019) 21(6):593-602. doi: 10.1016/j.jcyt.2019.03.003

50. Shi X, Zhang D, Li F, Zhang Z, Wang S, Xuan Y, et al. Targeting Glycosylation of PD-1 to Enhance CAR-T Cell Cytotoxicity. J Hematol Oncol (2019) 12 (1):127. doi: 10.1186/s13045-019-0831-5

51. Yin Y, Boesteanu AC, Binder ZA, Xu C, Reid RA, Rodriguez JL, et al. Checkpoint Blockade Reverses Anergy in IL-13R $\alpha 2$ Humanized scFv-Based Car T Cells to Treat Murine and Canine Gliomas. Mol Ther Oncolytics (2018) 11:20-38. doi: 10.1016/j.omto.2018.08.002

52. He X, Feng Z, Ma J, Ling S, Cao Y, Gurung B, et al. Bispecific and Split CAR T Cells Targeting CD13 and TIM3 Eradicate Acute Myeloid Leukemia. Blood (2020) 135(10):713-23. doi: 10.1182/blood.2019002779

53. Boice M, Salloum D, Mourcin F, Sanghvi V, Amin R, Oricchio E, et al. Loss of the HVEM Tumor Suppressor in Lymphoma and Restoration by Modified Car-T Cells. Cell (2016) 167(2):405-18. doi: 10.1016/j.cell.2016.08.032

54. Grosser R, Cherkassky L, Chintala N, Adusumilli PS. Combination Immunotherapy With CAR T Cells and Checkpoint Blockade for the Treatment of Solid Tumors. Cancer Cell (2019) 36(5):471-82. doi: 10.1016/ j.ccell.2019.09.006

55. Parriott G, Deal K, Crean S, Richardson E, Nylen E, Barber A. T-Cells Expressing a chimeric-PD1-Dap10-CD3zeta Receptor Reduce Tumour Burden in Multiple Murine Syngeneic Models of Solid Cancer. Immunology (2020) 160(3):280-94. doi: 10.1111/imm.13187

56. Rafiq S, Yeku OO, Jackson HJ, Purdon TJ, van Leeuwen DG, Drakes DJ, et al. Targeted Delivery of a PD-1-blocking scFv by CAR-T Cells Enhances AntiTumor Efficacy. vivo Nat Biotechnol (2018) 36(9):847-56. doi: 10.1038/ nbt. 4195

57. Eyquem J, Mansilla-Soto J, Giavridis T, van der Stegen SJ, Hamieh M, Cunanan KM, et al. Targeting a CAR to the TRAC Locus With CRISPR/ Cas9 Enhances Tumour Rejection. Nature (2017) 543(7643):113-7. doi: 10.1038/nature21405

58. Choi BD, Yu X, Castano AP, Darr H, Henderson DB, Bouffard AA, et al. Crispr-Cas9 Disruption of PD-1 Enhances Activity of Universal EGFRvIII Car T Cells in a Preclinical Model of Human Glioblastoma. J Immunother Cancer (2019) 7(1):304. doi: 10.1186/s40425-019-0806-7

59. Hu B, Zou Y, Zhang L, Tang J, Niedermann G, Firat E, et al. Nucleofection With Plasmid DNA for CRISPR/Cas9-Mediated Inactivation of Programmed Cell Death Protein 1 in CD133-Specific Car T Cells. Hum Gene Ther (2019) 30 (4):446-58. doi: 10.1089/hum.2017.234

60. Hamieh M, Dobrin A, Cabriolu A, van der Stegen SJC, Giavridis T, MansillaSoto J, et al. Car T Cell Trogocytosis and Cooperative Killing Regulate Tumour Antigen Escape. Nature (2019) 568(7750):112-6. doi: 10.1038/ s41586-019-1054-1

61. Greaves M. Evolutionary Determinants of Cancer. Cancer Discovery (2015) 5 (8):806-20. doi: 10.1158/2159-8290.Cd-15-0439

62. Rosenthal J, Naqvi AS, Luo M, Wertheim G, Paessler M, Thomas-Tikhonenko A, et al. Heterogeneity of Surface CD19 and CD22 Expression in B Lymphoblastic Leukemia. Am J Hematol (2018) 93(11):E352-e5. doi: 10.1002/ajh.25235

63. Orlando EJ, Han X, Tribouley C, Wood PA, Leary RJ, Riester M, et al. Genetic Mechanisms of Target Antigen Loss in CAR19 Therapy of Acute Lymphoblastic Leukemia. Nat Med (2018) 24(10):1504-6. doi: 10.1038/ s41591-018-0146-z

64. Ramakrishna S, Highfill SL, Walsh Z, Nguyen SM, Lei H, Shern JF, et al. Modulation of Target Antigen Density Improves CAR T-Cell Functionality and Persistence. Clin Cancer Res (2019) 25(17):5329-41. doi: 10.1158/10780432.Ccr-18-3784

65. Drent E, Poels R, Ruiter R, van de Donk N, Zweegman S, Yuan H, et al. Combined CD28 and 4-1BB Costimulation Potentiates Affinity-tuned Chimeric Antigen Receptor-Engineered T Cells. Clin Cancer Res (2019) 25 (13):4014-25. doi: 10.1158/1078-0432.Ccr-18-2559 
66. Majzner RG, Rietberg SP, Sotillo E, Dong R, Vachharajani VT, Labanieh L, et al. Tuning the Antigen Density Requirement for CAR T-Cell Activity. Cancer Discovery (2020) 10(5):702-23. doi: 10.1158/2159-8290.Cd-19-0945

67. Adachi K, Kano Y, Nagai T, Okuyama N, Sakoda Y, Tamada K. IL-7 and CCL19 Expression in CAR-T Cells Improves Immune Cell Infiltration and CAR-T Cell Survival in the Tumor. Nat Biotechnol (2018) 36(4):346-51. doi: $10.1038 /$ nbt.4086

68. Almåsbak H, Rian E, Hoel HJ, Pulè M, Wälchli S, Kvalheim G, et al. Transiently Redirected T Cells for Adoptive Transfer. Cytotherapy (2011) 13(5):629-40. doi: 10.3109/14653249.2010.542461

69. Maus MV, Haas AR, Beatty GL, Albelda SM, Levine BL, Liu X, et al. T Cells Expressing Chimeric Antigen Receptors can Cause Anaphylaxis in Humans. Cancer Immunol Res (2013) 1(1):26-31. doi: 10.1158/2326-6066.Cir-13-0006

70. Jones BS, Lamb LS, Goldman F, Di Stasi A. Improving the Safety of Cell Therapy Products by Suicide Gene Transfer. Front Pharmacol (2014) 5:254. doi: 10.3389/fphar.2014.00254

71. Moolten FL. Tumor Chemosensitivity Conferred by Inserted Herpes Thymidine Kinase Genes: Paradigm for a Prospective Cancer Control Strategy. Cancer Res (1986) 46(10):5276-81.

72. Tiberghien P, Ferrand C, Lioure B, Milpied N, Angonin R, Deconinck E, et al. Administration of Herpes Simplex-Thymidine Kinase-Expressing Donor T Cells With a T-cell-depleted Allogeneic Marrow Graft. Blood (2001) 97(1):6372. doi: 10.1182/blood.v97.1.63

73. Gargett T, Brown MP. The Inducible Caspase-9 Suicide Gene System as a "Safety Switch" to Limit on-Target, Off-Tumor Toxicities of Chimeric Antigen Receptor T Cells. Front Pharmacol (2014) 5:235. doi: 10.3389/fphar.2014.00235

74. Tasian SK, Kenderian SS, Shen F, Ruella M, Shestova O, Kozlowski M, et al. Optimized Depletion of Chimeric Antigen Receptor T Cells in Murine Xenograft Models of Human Acute Myeloid Leukemia. Blood (2017) 129 (17):2395-407. doi: 10.1182/blood-2016-08-736041

75. Paszkiewicz PJ, Fräßle SP, Srivastava S, Sommermeyer D, Hudecek M, Drexler I, et al. Targeted Antibody-Mediated Depletion of Murine CD19 Car T Cells Permanently Reverses B Cell Aplasia. J Clin Invest (2016) 126(11):4262-72. doi: $10.1172 /$ jci84813

76. Wu CY, Roybal KT, Puchner EM, Onuffer J, Lim WA. Remote Control of Therapeutic T Cells Through a Small Molecule-Gated Chimeric Receptor. Sci (New York NY) (2015) 350(6258):aab4077. doi: 10.1126/science.aab4077

77. Juillerat A, Marechal A, Filhol JM, Valton J, Duclert A, Poirot L, et al. Design of Chimeric Antigen Receptors With Integrated Controllable Transient Functions. Sci Rep (2016) 6:18950. doi: 10.1038/srep18950

78. Fedorov VD, Themeli M, Sadelain M. Pd-1- and CTLA-4-based Inhibitory Chimeric Antigen Receptors (iCARs) Divert Off-Target Immunotherapy Responses. Sci Trans Med (2013) 5(215):215ra172. doi: 10.1126/scitranslmed. 3006597

79. Reinhard K, Rengstl B, Oehm P, Michel K, Billmeier A, Hayduk N, et al. An RNA Vaccine Drives Expansion and Efficacy of claudin-CAR-T Cells Against Solid Tumors. Sci (New York NY) (2020) 367(6476):446-53. doi: 10.1126/science.aay5967

80. Minn I, Rowe SP, Pomper MG. Enhancing CAR T-Cell Therapy Through Cellular Imaging and Radiotherapy. Lancet Oncol (2019) 20(8):e443-e51. doi: 10.1016/s1470-2045(19)30461-9
81. Feng K, Liu Y, Guo Y, Qiu J, Wu Z, Dai H, et al. Phase I Study of Chimeric Antigen Receptor Modified T Cells in Treating HER2-positive Advanced Biliary Tract Cancers and Pancreatic Cancers. Protein Cell (2018) 9(10):83847. doi: 10.1007/s13238-017-0440-4

82. Porter CE, Rosewell Shaw A, Jung Y, Yip T, Castro PD, Sandulache VC, et al. Oncolytic Adenovirus Armed With BiTE, Cytokine, and Checkpoint Inhibitor Enables Car T Cells to Control the Growth of Heterogeneous Tumors. Mol Ther (2020) 28(5):1251-62. doi: 10.1016/j.ymthe.2020.02.016

83. Chen Q, Hu Q, Dukhovlinova E, Chen G, Ahn S, Wang C, et al. Photothermal Therapy Promotes Tumor Infiltration and Antitumor Activity of CAR T Cells. Adv Mater (Deerfield Beach Fla) (2019) 31(23):e1900192. doi: 10.1002/ adma.201900192

84. Ma W, Zhu D, Li J, Chen X, Xie W, Jiang X, et al. Coating Biomimetic Nanoparticles With Chimeric Antigen Receptor T Cell-Membrane Provides High Specificity for Hepatocellular Carcinoma Photothermal Therapy Treatment. Theranostics (2020) 10(3):1281-95. doi: 10.7150/thno.40291

85. Hernandez I, Prasad V, Gellad WF. Total Costs of Chimeric Antigen Receptor T-Cell Immunotherapy. JAMA Oncol (2018) 4(7):994-6. doi: 10.1001/ jamaoncol.2018.0977

86. Castella M, Boronat A, Martín-Ibáñez R, Rodríguez V, Suñé G, Caballero M, et al. Development of a Novel Anti-Cd19 Chimeric Antigen Receptor: A Paradigm for an Affordable Car T Cell Production At Academic Institutions. Mol Ther Methods Clin Dev (2019) 12:134-44. doi: 10.1016/j.omtm. 2018.11.010

87. Mock U, Nickolay L, Philip B, Cheung GW, Zhan H, Johnston ICD, et al. Automated Manufacturing of Chimeric Antigen Receptor T Cells for Adoptive Immunotherapy Using CliniMACS Prodigy. Cytotherapy (2016) 18(8):1002-11. doi: 10.1016/j.jcyt.2016.05.009

88. Hung CF, Xu X, Li L, Ma Y, Jin Q, Viley A, et al. Development of Anti-Human Mesothelin-Targeted Chimeric Antigen Receptor Messenger RNATransfected Peripheral Blood Lymphocytes for Ovarian Cancer Therapy. Hum Gene Ther (2018) 29(5):614-25. doi: 10.1089/hum.2017.080

89. Ghassemi S, Nunez-Cruz S, O'Connor RS, Fraietta JA, Patel PR, Scholler J, et al. Reducing Ex Vivo Culture Improves the Antileukemic Activity of Chimeric Antigen Receptor (Car) T Cells. Cancer Immunol Res (2018) 6 (9):1100-9. doi: 10.1158/2326-6066.Cir-17-0405

90. Richters MM, Xia H, Campbell KM, Gillanders WE, Griffith OL, Griffith M. Best Practices for Bioinformatic Characterization of Neoantigens for Clinical Utility. Genome Med (2019) 11(1):56. doi: 10.1186/s13073-019-0666-2

Conflict of Interest: The authors declare that the research was conducted in the absence of any commercial or financial relationships that could be construed as a potential conflict of interest.

Copyright (๑) 2021 Miao, Zhang, Ren, Tang and Li. This is an open-access article distributed under the terms of the Creative Commons Attribution License (CC BY). The use, distribution or reproduction in other forums is permitted, provided the original author(s) and the copyright owner(s) are credited and that the original publication in this journal is cited, in accordance with accepted academic practice. No use, distribution or reproduction is permitted which does not comply with these terms. 